\title{
ON A PROBLEM OF S. ULAM
}

\section{GILLES FOURNIER}

Abstract. Giving a negative answer to a problem of S. Ulam, the author provides an example of two noncomplete subspaces of $\boldsymbol{R}$ which are not isometric but whose squares are isometric.

In his book [1], S. Ulam formulated this problem:

"If $A$ and $B$ are metric spaces, then $A^{2}$ and $B^{2}$ may also be regarded as metric spaces, the metric of a product space $A^{2}$ being defined, for example, by $\rho\left(\left(a_{1}, a_{2}\right),\left(a_{3}, a_{4}\right)\right)=\left[\rho^{2}\left(a_{1}, a_{3}\right)+\rho^{2}\left(a_{2}, a_{4}\right)\right]^{1 / 2}$.

Does isometry of $A^{2}$ and $B^{2}$ imply that of $A$ and $B$ ?"

In this paper, we will give a negative answer to this question, producing a simple example of two nonisometric spaces $X$ and $Y$ such that the metric products $X^{2}$ and $Y^{2}$ are isometric.

Let $X=Q \subseteq R$, where $Q$ is the set of rational numbers, and let $Y=\{p \sqrt{ } 2 \mid p \in Q\} \subseteq R$. $X$ and $Y$ are metric subspaces of $R$, and $X$ is not isometric to $Y$. If $f$ were an isometry from $X$ to $Y$, then

$$
1=d(0,1)=d(f(0), f(1))=d(p \sqrt{ } 2, q \sqrt{ } 2)=\sqrt{ } 2|p-q|
$$

and so $\sqrt{ } 2=(|p-q|)^{-1} \in \mathbb{Q}$, which is a contradiction.

However, $X^{2}$ is isometric to $Y^{2}$. Considering these two spaces as metric subspaces of $R^{2}$, we study $\theta: R^{2} \rightarrow R^{2}$ where $\theta(x, y)$ $=((x-y) \sqrt{ } 2 / 2,(x+y) \sqrt{ } 2 / 2)$, the rotation by an angle of $\pi / 4$ and therefore an isometry of $R^{2}$ on to itself.

$\theta\left(X^{2}\right)=Y^{2}$, for $\theta\left(X^{2}\right) \subseteq Y^{2}:(p, q) \in X^{2}$ implies $\theta(p, q)=((p-q) \sqrt{ } 2 / 2$, $(p+q) \sqrt{ } 2 / 2) \in Y^{2}$, because $(p-q) / 2$ and $(p+q) / 2$ are rational; and $\theta\left(X^{2}\right) \supseteq Y^{2}:(p \sqrt{ } 2, q \sqrt{ } 2) \in Y^{2}$ is the image of $(p+q, q-p)$, an element of $X^{2}$.

The contraction $g$ of $\theta$ to the pair $\left(X^{2}, Y^{2}\right)$ (i.e. $g: X^{2} \rightarrow Y^{2}$ where $g(p, q)=\theta(p, q))$ is a distance-preserving bijection, and thus an isometry from $X^{2}$ to $Y^{2}$.

Notice that, in this example, $X$ and $Y$ are not complete metric spaces; so the following question may be raised: if $A$ and $B$ are complete metric spaces, does isometry of $A^{2}$ and $B^{2}$ imply that of $A$ and $B$ ?

\section{REFERENCES}

1. S. Ulam, $A$ collection of mathematical problems, Interscience Tracts in Pure and Appl. Math., no. 8, Interscience, New York, 1960. MR 22 \#10884.

Université de Montréal, Montréal, Québec, Canada

Received by the editors November 17, 1970.

AMS 1970 subject classifications. Primary 54E40; Secondary 54G20, 54-01.

Key words and phrases. Metric space, isometry, product of metric spaces. 